\title{
New results in software model checking and analysis
}

\author{
Corina S. Păsăreanu
}

Published online: 22 October 2010

(C) Springer-Verlag 2010

Modern software, which often involves complex concurrent computations and operates in an uncertain environment, must be highly reliable and secure. Commonly used techniques for addressing the reliability and safety of modern software systems include model checking and testing. Testing is widely used but it usually involves manual effort and it is ill-suited for finding concurrency errors. Model checking [3], on the other hand, has shown great promise in finding subtle program errors in a completely automated way.

Given a (model of a) system and a property, model checking systematically enumerates, explicitly or symbolically, all the (reachable) system configurations and it checks if they conform with the property. The result of model checking is either "true", if the property holds, or "false" if the property does not hold; in the latter case the model checking procedure also provides a detailed counter-example trace that leads to the property violation. Properties of interest include absence of deadlocks and data races in concurrent programs, or more general assertions and temporal logic formulae. Such formulae encode the expected behavior of the system in terms of safety and liveness, as well as timed, probabilistic or security properties.

To ensure that the model checking terminates, some form of abstraction is usually necessary to reduce the large search space for the original system into a smaller one that is more amenable for verification. Alternately, model checking can be used as an effective bug-finding technique, and the detailed counter-example traces provided can help debugging the discovered errors. The number of possible system configurations that needs to be explored is very large for most realistic practical applications. Consequently there has been

C. S. Păsăreanu ( $\varangle)$

Carnegie Mellon University/NASA Ames Research Center,

Moffett Field, CA 94035, USA

e-mail: corina.s.pasareanu@nasa.gov continuous effort spent over the years to address this scalability problem.

The articles enclosed here describe new model checking techniques, supported by robust and scalable tools, for the automated analysis of modern software systems. The articles have been carefully reviewed and they are based on papers that were considered to be among the best at the SPIN 2009 model checking workshop [11]. The topics addressed range from probabilistic model checking and parallelization techniques for improved scalability to data race detection, symbolic analysis and model checking for security.

The first article [5], presents an effective technique for computing the probability of reaching a given set of states in a parametric Markov model. Such models can be used to reason about quantitative properties in systems where certain aspects are not fixed, but rather depend on parameters. Previous work [4] has suggested to convert the Markov chain into a finite automaton, equivalent to a regular expression. The expression can be evaluated to a closed form function representing the reachability probability. The bottleneck of the approach lies in the growth of the regular expression with the number of states. The authors propose to remedy the problem by intertwining the regular expression computation with its evaluation. This results into a practical method that has been implemented in the PARAM tool and has been demonstrated experimentally on network protocols.

The second article [2] is also concerned with probabilistic reasoning, in the context of the PRISM model checking tool [9], where the satisfaction of desired properties is quantified with some probability. The authors propose algorithms for parallel probabilistic model checking using general purpose graphic processing units. The proposed improvements target the numerical computations of the traditional sequential algorithms since these computations can be parallelized efficiently on graphic processors. The parallel algorithms have 
been implemented in the PRISM model checker and have been evaluated on several case studies, showing significant speed-up.

The third article [8] addresses the problem of verifying data consistency in concurrent Java programs. The work targets data races caused by inconsistent accesses to multiple fields of an object-the so-called atomic-set serializability problem. Previous work used abstraction techniques to translate a concurrent Java program into an EML program, a modeling language based on push-down systems and a finite set of re-entrant locks, and used only a semi-decision procedure to check the program. The present article extends that work by describing a full decision procedure for verifying data consistency, i.e., atomic-set serializability, of an EML program. The procedure has been implemented and it has been applied to detect both single-location and multi-location data races in models of concurrent Java programs.

The fourth article [10] presents a generic technique for creating the basic primitives used in symbolic program analysis: forward symbolic evaluation, weakest liberal precondition, and symbolic composition. Using this technique, one can automatically generate an implementation of a (forward or backward) symbolic program execution at the cost of writing only the specification of the concrete program semanticsin the form of an interpreter for the language of interest. The technique can be used for programming languages with pointers and arithmetic operations. The technique has been implemented and it has been used to generate symbolicanalysis primitives for the $\mathrm{x} 86$ and PowerPC instruction sets. The symbolic analysis generated with the generic technique presented here can be used in software model checking tools such as SLAM [1] and Blast [6], as well as in other automated bug-finding tools that rely on symbolic reasoning $[14,15]$

Finally, the fifth article [13] presents an application of the SPIN model checker [7] to checking signature specifications. Signatures are matching rules that are used in intrusion detection systems when searching for attack traces in the recorded audit data based on pre-defined patterns. Intrusion detection systems are one of the most important means to protect information technology systems [12]. The effectiveness of an intrusion detection system depends on the adequacy of the signatures, which are usually defined empirically. Modeling a new signature is time-consuming and error-prone; consequently the modeled signature needs to be tested carefully. In this article, the authors present an approach to automatically checking signature specifications using the SPIN model checker. The signatures are modeled in the specification language EDL (a variant of Petri-nets) and then translated into
PROMELA, the input language of the SPIN model checking tool. SPIN is used to find specification errors, which are modeled using linear temporal logic.

In conclusion, the articles enclosed here describe new results in software model checking and analysis. The presented techniques are most useful at finding subtle and costly errors that can not be found with traditional testing alone. The techniques have been implemented in robust tools and therefore show good promise for adoption in industry.

\section{References}

1. Ball, T., Majumdar, R., Millstein, T., Rajamani, S.: Automatic predicate abstraction of $\mathrm{C}$ programs. In: Proceedings of the Conference on Programming Language Design and Implementation (PLDI 2001), Snowbird, Utah (2001)

2. Bosnacki, D., Edelkamp, S., Sulewski, D., Wijs, A.: Parallel probabilistic model checking on general purpose graphics processors. Int. J. Softw. Tools Technol. Transf. (this volume)

3. Clarke, E.M., Grumberg, O., Peled, D.A.: Model Checking. MIT Press, Cambridge (2000)

4. Daws, C.: Symbolic and parametric model checking of discretetime markov chains. In: Proc. ICTAC, pp. 280-294 (2004)

5. Hahn, E.M., Hermanns, H., Zhang, L.: Probabilistic reachability for parametric markov models. Int. J. Softw. Tools Technol. Transf. (this volume)

6. Henzinger, T.A., Jhala R., Majumdar, R., Sutre, G.: Lazy abstraction. In: Proceedings of the 29th Annual Symposium on Principles of Programming Languages (POPL) (2002)

7. Holzmann, G.J.: The Spin Model Checker: Primer and Reference Manual. Addison-Wesley, Reading (2003)

8. Kidd, N., Reps, T., Touili, T., Lammich, P.: A decision procedure for detecting atomicity violations for communicating processes with locks. Int. J. Softw. Tools Technol. Transf. (this volume)

9. Kwiatkowska, M., Norman, G., Parker, D.: Prism: probabilistic model checking for performance and reliability analysis. ACM SIGMETRICS Perform. Eval. Rev. 36(4), 40-45 (2009)

10. Lim, J., Lal, A., Reps, T.: Symbolic analysis via semantic reinterpretation. Int. J. Softw. Tools Technol. Transf. (this volume)

11. Păsăreanu, C.S. (ed.): Model checking software. In: Proceedings of the 16th International SPIN Workshop, Grenoble, France, June 26-28, 2009. Lecture Notes in Computer Science, vol. 5578. Springer, Berlin (2009)

12. Scarfone, K., Mell, P.: Guide to intrusion detection and prevention systems (IDPS). Computer Security Resource Center (National Institute of Standards and Technology) (800-94) (2007)

13. Schmerl, S., Vogel, M., Konig, H.: Using model checking to identify errors in intrusion detection signatures. Int. J. Softw. Tools Technol. Transf. (this volume)

14. Sen, K., Marinov, D., Agha, G.: CUTE: a concolic unit testing engine for C. In: Proceedings of ESEC/FSE'05 (2005)

15. Xie, Y., Chou, A., Engler, D.: ARCHER: using symbolic, path-sensitive analysis to detect memory access errors. In: Proceedings of ESEC/FSE'03 (2003) 